\title{
¿Cómo trabajar con modelos en clase de ciencias? Ideas de los maestros en formación sobre el papel de la modelización en la enseñanza de las ciencias
}

Beatriz Bravo, Beatriz Mazas, Ana Aragüés, María José Sáez y Ana de Echave. Universidad de Zaragoza

Recepción: 12 de abril de 2015 | Revisión: 30 de mayo de 2015 | Aceptación/Publicación: 24 de julio de 2015

Correspondencia: bbtorija@unizar.es | bmazas@unizar.es | araguesd@unizar.es | msaezbo@unizar.es | aechave@unizar.es

Resumen: En este trabajo se examinan las ideas de 201 maestros de Educación Primaria en formación sobre el uso de los modelos en las clases de ciencias. Para ello, se elaboró un cuestionario con cinco preguntas abiertas, de las cuales en este estudio se analiza la siguiente ¿Cómo consideras que se ha de trabajar con modelos en la enseñanza de las ciencias? Los futuros maestros debían escoger entre siete ítems que señalaban distintas formas de trabajar con modelos y justificar su elección. Cinco de ellos se relacionaban con funciones propias de la modelización y los otros dos consideraban los modelos como objetos de trasmisión de conocimiento. Para el análisis se tuvo en cuenta tanto los ítems elegidos como la justificación realizada. Los resultados muestran que a pesar de que reconocen la importancia de la observación, comparación y discusión de los modelos en el aula, los maestros tienen dificultades para justificar a qué dimensión de la modelización contribuyen estas acciones. Tan solo en uno de los ítems (presentación de modelos que resumen la información), el número de justificaciones con información relevante supera al número de justificaciones que parafrasean el ítem o incluyen información irrelevante. Una implicación derivada de este estudio es la necesidad de diseñar actividades de formación en que se trabajen tanto ejemplos concretos de modelos teóricos, como la relevancia de estos en la generación y validación del conocimiento científico.

Palabras clave: Formación de profesorado | Modelización | Prácticas científicas | Modelo científico

HOW TO WORK WITH MODELS IN SCIENCE CLASS? PRE-SERVICE TEACHERS' IDEAS ABOUT THE ROLE OF MODELLING IN SCIENCE EDUCATION

\begin{abstract}
In this work we examine the ideas of 201 preservice primary teachers about the use of models in science classrooms. To do so, a questionnaire of five open-items was designed. In this study we focus on how do you think you should work with models in science education? The preservice teachers should choose among seven items addressing different ways to work with models in science classroom. Five out of seven were related to modelling's functions and the other two with the use of models as objects of communication. For the analysis, we took into account both the item chosen for the participants and the justification provided by them. The results show that although the preservice teachers recognise the importance of observing, comparing and discussing models in classroom, they encounter difficulties justifying how these actions could contribute to each dimension of modelling. Only in one item, the presentation of models as summary of information, the number of justifications with relevant information surpassed the justifications that rephrased the item or included irrelevant information. One implication that arises from this study is the need to design specific training activities focus on both working with theoretical models and considering their relevance in the generation and validation of scientific knowledge.
\end{abstract}

Keywords: Teachers Training | Modelling | Scientific Practices | Scientific Model

Agradecimientos: Al proyecto de innovación docente con código PIIDUZ_13_087, perteneciente a la Universidad de Zaragoza. A los alumnos del grado de Magisterio de Educación Primaria.

\section{Introducción}

La modelización entendida como la construcción, uso y evaluación de modelos ha sido reconocida como un proceso clave tanto en el desarrollo del conocimiento científico (Gilbert y Boulter, 1998) como en la enseñanza de las ciencias (Acher, Arcá y SanMartí, 2007). Diversos estudios han mostrado que trabajar con actividades que promueven la modelización favorece no solo una mejora en la adquisición del conocimiento científico (Jonassen, 2004; Megalakaki y Tiberghien, 2011; Oh y Oh, 2011), sino que proporciona oportunidades para realizar predicciones, guiar investigaciones, justificar resultados, y comunicarlos de forma sencilla (Gilbert y Boulter, 1998). A su vez, ayuda al profesorado a conocer los modelos mentales de su alumnado y a comprobar cómo cambian y evolucionan en el tiempo (Mendonça y Justi, 2011). Pero para poder conseguirlo es necesario promover ambientes de aprendizaje en que los alumnos sean quienes construyan sus propios modelos, los utilicen y 
evalúen su validez. Sin embargo, autores como Grosslight et al. (1991) señalan que en las aulas los modelos son utilizados como meros objetos de conocimiento, cuyo objetivo principal es ayudar o facilitar la comprensión del alumnado. Una de las razones por las que predomina este uso de los modelos en las aulas podría relacionarse con las actitudes de los profesores hacia las ciencias y hacia su enseñanza. En concreto, las creencias acerca de qué son los modelos, para qué se utilizan y cómo se han de trabajar en el aula, condicionan la forma de presentárselos al alumnado (Justi y Gilbert, 2003). La mayoría de estudios realizados con profesorado en activo han mostrado que este posee un conocimiento limitado e inconsistente en cuanto a la naturaleza de los modelos y a su función en la generación y evaluación del conocimiento científico (ver revisión de Oh y Oh, 2011). Justi y Gilbert (2003) mostraron que la mayoría del profesorado identificaba los modelos como estructuras o procesos del mundo real, cuya función principal era la de describir y explicar los fenómenos de la naturaleza; sin reconocer otras como la predicción, la formulación de hipótesis o la comunicación de resultados. Resultados similares fueron encontrados por van Driel y Verloop (1999) e Isla y Pesa (2003). Son pocos los estudios que han explorado la visión de los futuros docentes sobre esta práctica científica. Consideramos necesario realizar trabajos dirigidos a conocer cómo piensan estos futuros docentes, con el objeto de diseñar programas adecuados a sus necesidades. Por todo lo anterior, se presenta un estudio preliminar cuyo objetivo es examinar cómo consideran los futuros maestros de Educación Primaria que se han de utilizar los modelos en la enseñanza y el aprendizaje de las ciencias.

\section{Métodos}

Los participantes fueron 201 estudiantes del grado de Maestro de Educación Primaria, pertenecientes a las tres facultades de educación de la Universidad de Zaragoza. Todos se encontraban cursando la asignatura de Didáctica del Medio Biológico y Geológico, y ninguno había trabajado previamente la modelización de forma explícita durante su formación anterior.

El cuestionario que se administró se componía de cinco preguntas abiertas con las que se pretendía conocer la visión del futuro profesorado acerca de qué son los modelos científicos, qué funciones y características tienen, y cómo han de trabajarse en el aula. La pregunta analizada fue: ¿Cómo consideras que se ha de trabajar con los modelos en la enseñanza de las ciencias? Escoge las opciones que consideras adecuadas y justifica porqué. Junto con la pregunta se proporcionaron siete ítems, cinco correspondían a las distintas dimensiones de la modelización, y los otros dos se relacionaban con el uso de los modelos como transmisión de conocimiento (van Driel y Verloop, 1999) (tabla 1).

\begin{tabular}{|c|c|c|}
\hline \multicolumn{3}{|r|}{$\begin{array}{c}\text { Tabla } 1 \\
\text { Relación uso de los modelos-dimensiones de la modelización }\end{array}$} \\
\hline Modelización & ítem & Usos de los modelos \\
\hline Construcción & $B$ & $\begin{array}{l}\text { Se debe promover la construcción de modelos a partir de la observación de un fenómeno } \\
\text { determinado. }\end{array}$ \\
\hline Uso & G & $\begin{array}{l}\text { Se deben utilizar los modelos para la resolución de problemas, realizando predicciones y } \\
\text { explicaciones en base a estos. } \\
\text { Se deben utilizar los modelos para facilitar la transferencia de lo aprendido a otros } \\
\text { contextos. }\end{array}$ \\
\hline Evaluación & D & $\begin{array}{l}\text { Se deben comparar y discutir distintos modelos de un mismo fenómeno reconociendo sus } \\
\text { limitaciones. } \\
\text { Se deben revisar modelos en base a las pruebas disponibles }\end{array}$ \\
\hline $\begin{array}{l}\text { Transmisión de } \\
\text { conocimiento }\end{array}$ & & $\begin{array}{l}\text { Se deben proporcionar modelos que resuman la información que queremos presentar a } \\
\text { alumnado. } \\
\text { Se deben presentar modelos adecuados que ayuden a explicar un fenómeno y faciliten su } \\
\text { comprensión. }\end{array}$ \\
\hline
\end{tabular}


El cuestionario se diseñó en base a los realizados en los estudios de Justi y Gilbert (2003) y van Driel y Verloop (1999) y se pasó a una muestra de 22 alumnos del grado de Magisterio y a cuatro docentes en ejercicio para su validación. Se modificaron aquellas preguntas que presentaban dificultades en su comprensión.

Para el análisis de las respuestas, en primer lugar se estimaron las frecuencias con las que habían sido escogidos cada uno de los ítems, y en segundo se analizaron las justificaciones proporcionadas. Para examinar las justificaciones de los futuros maestros, nos basamos en el análisis del contenido, distinguiendo entre si aportaban información que relacionara el ítem con la dimensión de la modelización que se trabajaba, si lo parafraseaban o si aportaban información no relevante para la tarea. Se presenta un ejemplo con el ítem D para clarificar la realización del análisis. Las respuestas del profesorado son textuales, manteniendo tanto faltas de ortografía como los errores de puntuación.

Los participantes debían relacionar el ítem $\mathrm{D}$ con la dimensión de la evaluación de modelos. En su justificación debían considerar la importancia de comparar distintos modelos con el fin de que sus alumnos reconozcan que no hay un solo modelo válido para explicar un fenómeno, y que dependiendo del objetivo del estudio se pueden utilizar uno u otro. También podrían añadir a esta idea la relacionada con el cambio en los modelos, incidiendo en que no son algo inmutable sino que van modificándose al ser revisados por la comunidad científica. Cualquier justificación que hiciera referencia a alguna de estas ideas se considera como información relevante. Un ejemplo de esta categoría es MT30 "No un único modelo es válido para la resolución de un problema pueden existir varios y entre ellos se debe seleccionar el que mejor se adapta al problema y a la posible solución".

En la categoría en la que parafrasean el ítem se incluyen todas aquellas respuestas que aunque cambien el orden de palabras o las palabras en sí, la información que aportan es la misma, un ejemplo lo encontramos en MZ6 "es importante comparar y ver las limitaciones de cada modelo para comprenderlos bien".

En relación a los que aportan información no relacionada directamente con el ítem, podemos encontrar justificaciones que apelan a su utilidad para aprender sin especificar por qué, MZ16 "comparar modelos puede ser muy útil para aprender" o la importancia de desarrollar un espíritu crítico, sin reconocer su contribución a la evaluación MH8 "de esta manera el alumno puede ser crítico con los modelos". Una vez definida cada una de las categorías, se analizaron todas las respuestas de los docentes siguiendo el proceso anterior.

\section{Resultados}

En este apartado en primer lugar se considera qué usos son los más escogidos por los futuros docentes y en segundo el tipo de justificaciones que proporcionan. En la tabla 2 se presentan los resultados para cada uno de los ítems. En una misma respuesta se podía hacer referencia a más de una función, por ello el número total de funciones escogidas $(n=613)$ es superior al número de participantes del estudio. 


\begin{tabular}{|c|c|c|}
\hline \multicolumn{3}{|c|}{$\begin{array}{c}\text { Tabla } 2 \\
\text { Uso de los modelos según los maestros en formación }\end{array}$} \\
\hline Ítems & Categorías & $\mathbf{N}^{0}$ \\
\hline Ítem A. & & $73(12 \%)$ \\
\hline & Justificaciones relevantes para el ítem & 11 \\
\hline & Parafrasean el ítem & 7 \\
\hline & Justificaciones generales o no relacionadas con el ítem & 55 \\
\hline Ítem B. & & $122(20 \%)$ \\
\hline & Justificaciones relevantes para el ítem & 30 \\
\hline & Parafrasean el ítem & 19 \\
\hline & Justificaciones generales o no relacionadas con el ítem & 73 \\
\hline Ítem C & & $39(6 \%)$ \\
\hline & Justificaciones relevantes para el ítem & 24 \\
\hline & Parafrasean el ítem & 4 \\
\hline & Justificaciones generales o no relacionadas con el ítem & 11 \\
\hline Ítem D & & $109(19 \%)$ \\
\hline & Justificaciones relevantes para el ítem & 40 \\
\hline & Parafrasean el ítem & 16 \\
\hline & Justificaciones generales o no relacionadas con el ítem & 53 \\
\hline Ítem E & & $87(14 \%)$ \\
\hline & Justificaciones relevantes para el ítem & 38 \\
\hline & Parafrasean el ítem & 11 \\
\hline & Justificación que aportan información relevante al ítem & 38 \\
\hline Ítem F & & $91(15 \%)$ \\
\hline & Justificaciones relevantes para el ítem & 23 \\
\hline & Parafrasean el ítem & 17 \\
\hline & Justificaciones generales o no relacionadas con el ítem & 51 \\
\hline Ítem G & & $74(14 \%)$ \\
\hline & Justificación que aportan información relevante al ítem & 13 \\
\hline & Parafrasea el ítem & 11 \\
\hline & Justificaciones generales o no relacionadas con el ítem & 50 \\
\hline Total & & $613(100 \%)$ \\
\hline
\end{tabular}

Como se muestra en la tabla, los usos más escogidos son: el ítem $B$, construcción de modelos a partir de la observación de un fenómeno, y el ítem $D$, discusión de distintos modelos de un mismo fenómeno reconociendo sus limitaciones, con una frecuencia de $20 \%$ y $19 \%$ respectivamente. Sin embargo, aunque los futuros maestros han sido capaces de reconocer la importancia de esta forma de trabajar con los modelos, encuentran dificultades para justificar en qué aspectos concretos contribuyen a la modelización. Por ejemplo, el ítem B lo deberían relacionar con la dimensión de construcción. En concreto, deberían señalar la importancia de que sean los alumnos quienes: consideren el tipo de fenómeno a estudiar, identifiquen sus componentes y cómo se relacionan, y en base a ellos construyan sus propios modelos. Sin embargo solo 30 de los 122 maestros que señalan esta opción aportan alguna información relacionada con la explicación anterior. Por ejemplo MZ42 "ya que si ellos mismos son los que piensan que está sucediendo por qué sucede qué interviene. El aprendizaje será más significativo".

El resto o proporcionan justificaciones generales o parafrasean el ítem. Entre las justificaciones generales destacan las que hacen referencia al método científico y las relacionadas con la adquisición de un aprendizaje significativo o el aumento de la motivación del alumnado. Un ejemplo es el de MH 50 "sí, ya que así es como funciona el método científico y los modelos científicos".

Este comportamiento también se observa en otras opciones escogidas en menor proporción como el ítem $\mathrm{A}$, utilización de modelos para la resolución de problemas. Solo 11 de 73 estudiantes de Magisterio son capaces de proporcionar alguna justificación válida. Al igual que en los anteriores la mayoría apelan a ideas como que el uso de modelos aumenta la participación del alumnado y su interés, facilita la 
comprensión del alumnado o promueve el aprendizaje significativo, pero sin especificar por qué.

Solo en el ítem $\mathrm{C}$, se deben proporcionar modelos que resuman la información que queremos presentar al alumnado, el número de justificaciones válidas supera a las otras dos, 24 frente a 15 de las 39 justificaciones proporcionadas. Esto nos lleva a considerar, que a pesar de ser la opción menos escogida por los futuros maestros, es con la que podrían están más familiarizados. Entre las justificaciones proporcionadas, encontramos las que señalan la importancia de proporcionar un conocimiento organizado para facilitar su comprensión MH43 "Ordenar y seleccionar la información es principal para no saturar o confundir a los alumnos", o las que hacen referencia a facilitar la comprensión al alumnado, MT36 "la información debe estar resumida y ser breve para comprenderla mejor". Resultados similares se han encontrado en trabajos como el de Isla y Pesa (2003).

\section{Conclusiones}

Los resultados obtenidos nos llevan a considerar que aunque los futuros maestros son capaces de escoger opciones relacionadas con prácticas propias de la modelización, tienen dificultad para justificar a qué dimensión de la modelización contribuyen y por qué es importante trabajarla en el aula. Como han mostrado estudios anteriores, este problema podría estar influenciado por una parte por cómo han trabajado ellos como estudiantes los modelos en sus aulas (Isla y Pesa, 2003), y por otra, con el limitado conocimiento que tienen la mayoría de los docentes acerca de las funciones de los modelos tanto en las ciencias como en su enseñanza (Justi y Gilbert, 2003).

Por todo ello, sugerimos la necesidad de realizar programas de formación específicos que combinen actividades de aprendizaje de ciencias, considerando por ejemplo qué conceptos se encuentran implicados en un modelo científico concreto y qué dificultades encuentran los alumnos en su adquisición, con actividades de aprendizaje sobre la ciencias y los procesos implicados. Una posible opción podría ser proporcionar al profesorado situaciones de aula en las que se trabajen modelos científicos y que, en base al modelo tratado y al proceso de modelización implicado, deban decidir cuál sería la más adecuada y por qué. Ya que de acuerdo con van Driel y Verloop (1999) una de las estrategias para favorecer que el profesorado adquiera una mejor comprensión de las ciencias como proceso, y no solo como producto, es reflexionar sobre situaciones que más tarde podrán utilizar con sus estudiantes.

\section{Referencias}

Acher, A., Arcá, M. \& SanMartí, N. (2007). Modeling as a teaching learning process for understanding materials: a case study in primary education. Science Education, 91, 398-418.

Gilbert, J. \& Boulter, C. (1998): Learning science through models and modelling. In B. Fraser \& K. Tobin (Eds.). International handbook of Science Education (pp. 53-66). Dordrecht; Kluwer.

Grosslight, L., Unger, C. \& Jay, E. (1991). Understanding models and their use in science: conceptions of middle and high school students and experts. Journal of Research in Science Teaching, 28(9), 799-822.

Isla, S. y Pesa, M. (2003). ¿Qué rol asignan los profesores de física de nivel medio a los modelos científicos y a las actividades de modelado? Enseñanza de las ciencias, número extra, 57-66.

Jonassen, D. H. (2004). Model building for conceptual change: using computers as cognitive tools, 4 Congres ETIIE, October, University of Athens.

Justi, R. \& Gilbert, J. (2003). Teachers' views on the nature of models. International Journal of Science Education, 25(11), 1369-1386. 
Megalakaki, O., \& Tiberghien, A. (2011). A qualitative approach of modelling activities for the notion of energy. Electronic Journal of Research in Educational Psychology, 9(1), 157-182

Mendonça, P. \& Justi, R. (2011). Contribution of the 'Model of Modelling' diagram to the learning of ionic bonding: Analysis of a case study. Research in Science Education, 41(4), 479-503.

Oh, P. \& Oh, S. (2011): What teachers of science need to know about models: an overview. International Journal of Science Education, 33(8), 1109-1130.

van Driel, J. \& Verloop, N. (1999). Teachers' knowledge of models and modelling in science. International Journal of Science Education, 21(11), 1141-1153. 